\title{
AN EMBEDDING THEOREM FOR COMMUTATIVE LATTICE-ORDERED DOMAINS
}

\author{
STUART A. STEINBERG
}

\begin{abstract}
In a recent paper Conrad and Dauns have shown that a finitely-rooted lattice-ordered field $R$, in which multiplication by a positive special element is a lattice homomorphism, can be embedded in a formal power series $l$-field with real coefficients, provided that the value group of $R$ is torsion-free. In this note it is shown that their theorem is true when $R$ is a commutative integral domain.
\end{abstract}

The proof of this theorem will be the same as the proof of Conrad and Dauns' result [3, Theorem III] once it has been established that a quotient ring of $R$ can be lattice-ordered. ${ }^{1}$ Their arguments do not use that $R$ is a field but only that the set of positive special elements of $R$ is a multiplicative group.

This paper represents a portion of the author's dissertation written at the University of Illinois at Urbana-Champaign under the direction of Professor Elliot C. Weinberg.

1. Extending the lattice order to a quotient ring. The following lemma may be thought of as a generalization of [3, Theorem I] (as may Lemma 2, also).

LEMMA 1. Let $R$ be an l-ring containing a positive invertible element $u$. Then right (left) multiplication by $u$ is a lattice homomorphism (equivalently, an l-automorphism of the underlying l-group of $R$ ) if and only if $u^{-1}$ is positive.

PRoOF. If $x \rightarrow x u$ is a lattice homomorphism, then ( $1 \vee 0) u=u \vee 0=u$; so $1>0$. Also, $\left(u^{-1} \vee 0\right) u=1 \vee 0=1$ implies $u^{-1} \vee 0=u^{-1}$; i.e., $u^{-1}>0$. Conversely, suppose that $u^{-1} \in R^{+}$. If $a$ is any positive element of $R$, then $x a \vee y a \leqq(x \vee y) a$. So,

$$
x \vee y=x u u^{-1} \vee y u u^{-1} \leqq(x u \vee y u) u^{-1} \leqq(x \vee y) u u^{-1}=x \vee y .
$$

Thus $(x \vee y) u=x u \vee y u$.

Received by the editors January 6, 1971.

AMS 1970 subject classifications. Primary 06A70, 16A08; Secondary 13G05, 06A60, 13J05.

Key words and phrases. Lattice-ordered ring, commutative domain, formal power series, classical ring of quotients, value preserving $l$-isomorphism, finitely-valued l-group, special element.

${ }^{1}$ The author would like to thank Professor Paul Conrad for pointing out a mistake in an earlier version of Theorem 2 .

(c) American Mathematical Society 1972 
Proposition 1. Let $R$ be an l-ring, and let $\Sigma$ be a multiplicative subset of positive regular elements. Suppose that $R$ is a right Ore ring with respect to $\Sigma$, and let $Q$ be its classical right quotient ring with respect to $\Sigma$. Then $Q$ can be made into an l-ring extension of $R$, in which the inverse of each element of $\Sigma$ is positive, exactly when left multiplication and right multiplication by each element of $\Sigma$ are lattice homomorphisms. If this is the case, the lattice order of $Q$ is uniquely determined by that of $R$.

Proof. Suppose that $x^{+} a=(x a)^{+}$and $a x^{+}=(a x)^{+}$for all $x \in R, a \in \Sigma$. Define $Q^{+}=\left\{q \in Q: q\right.$ has a representation of the form $q=x a^{-1}$ for some $\left.(x, a) \in R^{+} \times \Sigma\right\}$. We claim that $\left(Q, Q^{+}\right)$is an $l$-ring extension of $\left(R, R^{+}\right)$ and that $a \in \Sigma$ implies $a^{-1} \in Q^{+}$. We first note that if $q \in Q^{+}$and $q=y b^{-1}$, then $y \in R^{+}$. Suppose, then, that $x a^{-1}=y b^{-1}$ with $x \in R^{+}$. There exists $(c, d) \in R \times \Sigma$ such that $x c=y d$ and $a c=b d$. Then $0=(a c)^{-}=a c^{-}$implies that $c \in R^{+}$, since $a$ is regular. Therefore $0=(x c)^{-}=(y d)^{-}=y^{-} d$, so $y \in R^{+}$.

It is easily seen that $Q^{+}$is a positive cone for the ring $Q$ and that $Q^{+} \cap R=$ $R^{+}$. Note that the least upper bound of two elements in $R$ is also their least upper bound in $Q$. For suppose that $(q, x) \in Q \times R, q=y a^{-1}$, and $q \geqq\{x, 0\}$. Then $y \geqq\{x a, 0\}$; so $y \geqq(x a)^{+}=x^{+} a$ and $q \geqq x^{+}$. Note, also, that if $a \in \Sigma$, then $a^{-1}=a\left(a^{2}\right)^{-1} \in Q^{+}$.

If $q=x a^{-1} \in Q$, define $q^{*}=x^{+} a^{-1}$. Then $q^{*}$ is independent of the representation of $q$. For if $q=y b^{-1}$, then there exists $(c, d) \in R \times \Sigma$ such that $b c=a d$ and $y c=x d$. Since $a, b$, and $d$ are in $\Sigma, c \in R^{+}$and $c^{-1} \in Q^{+}$. Since right multiplication by $c$ is an order isomorphism of $Q$ onto $Q$, and thus preserves all existing sups, $y^{+} c=(y c)^{+}=(x d)^{+}=x^{+} d$. Thus $y^{+} b^{-1}=x^{+} a^{-1}$, and $q^{*}$ is well defined. It is now easily seen that $q^{*}=q \vee 0$.

The converse is immediate from Lemma 1 . For uniqueness, suppose that $(Q, P)$ is an $l$-ring extension of $\left(R, R^{+}\right)$such that $a \in \Sigma$ implies $a^{-1} \in P$. Then, clearly, $Q^{+} \subseteq P$. If $x a^{-1} \in P$, then $x=\left(x a^{-1}\right) a \in P \cap R=R^{+}$. So $P=R^{+}$.

We have essentially used Anderson's proof of the special case of the following corollary, i.e., that in which $\Sigma$ is the set of all regular elements of the unital $f$-ring $R[1$, Theorem 5.1], to prove Proposition 1.

COROLlARY. Let $R$ be a right Ore ring with respect to a multiplicative subset of regular elements $\Sigma$, and let $Q$ be its classical right quotient ring with respect to $\Sigma$. If $R$ is an f-ring, then $Q$ can be made into an f-ring extension of $R$ in exactly one way.

Proof. Let $\Sigma^{+}=\Sigma \cap R^{+}$. If $q \in Q$, then $q=x a^{-1}=x a\left(a^{2}\right)^{-1}$ and $a^{2} \in \Sigma^{+}$. Thus $Q$ is the right quotient ring of $R$ with respect to $\Sigma^{+}$. Now use Proposition 1. 
2. D-domains. A value of a nonzero element $g$ in an $l$-group is a convex $l$-subgroup that is maximal with respect to the exclusion of $g$. A special element of an $l$-group $G$ is an element that has exactly one value. Note that a special element is comparable to $0 . G$ is finitely-valued if each of its elements has only a finite number of values. For the remainder of this paper, by a special element we shall mean a positive special element. A basic element is a positive nonzero element $g$ for which the convex $l$ subgroup generated by $g, C(g)$, is totally ordered. It is known that a basic element is special. For the theory of special elements see [2]. The following lemma is an immediate consequence of Lemma 1.

LEMMA 2. Let $R$ be an l-ring containing an invertible element $u$ such that $u$ and $u^{-1}$ are both positive.

(a) If $g \in R$, then $g$ is special (basic) if and only if ug is special (basic).

(b) 1 is special (basic) if and only if $u$ is special (basic).

(c) $u$ is special (basic) if and only if $u^{-1}$ is special (basic).

An $l$-domain is an $l$-ring $R$ in which $R^{+} \backslash\{0\}$ is a multiplicatively closed subset [6].

COROLlary. If $R$ is an l-domain, then $u$ is basic.

Proof. $C(1)$, the convex $l$-subgroup of $R$ generated by 1 , is an $f$-ring since 1 is a strong order unit in $C(1)$. Since $C(1)$ is an $l$-domain, it is totally ordered.

This corollary is actually a generalization of the fact that a latticeordered division ring in which the inverse of every positive element is positive must be totally ordered. This is proven in [7, p. 199] for the commutative case.

By a $D$-domain we shall mean a commutative lattice-ordered ring $R$, without zero divisors, such that

(a) the set $S$ of special elements of $R$ is nonempty, and

(b) multiplication by an element of $S$ is a lattice homomorphism.

PROPOSITION 2. Let $R$ be a commutative lattice-ordered integral domain. Then $R$ is a $D$-domain if and only if

$$
S_{1}=\left\{0 \neq s \in R^{+}: \text {multiplication by } s \text { is a lattice homomorphism }\right\}
$$

is nonempty and $S=S_{1}$. In particular, the special elements in a D-domain form a multiplicatively closed subset.

Proof. If $R$ is a a $D$-domain, then $S \subseteq S_{1}$, so $S_{1}$ is nonempty. Clearly $S_{1}$ is multiplicatively closed. Let $Q$ be the ring of quotients of $R$ with respect to $S_{1}$. By Proposition $1, Q$ is an $l$-ring extension of $R$, and its 
positive cone is given by

$$
Q^{+}=\left\{a s^{-1} \in Q: a \in R^{+}, s \in S\right\} .
$$

If $s \in S_{1}$, then $s$ and $s^{-1}$ are in $Q^{+}$. By the previous corollary, $s$ is basic in $Q$; hence it is also basic in $R$. Thus $S=S_{1}$. The converse is trivial

In $[3$, Theorem I] it is shown that an $l$-field $R$ is a $D$-domain if and only if its set of special elements $S$ forms a multiplicative group. The following example shows that a finitely-rooted, commutative, lattice-ordered domain, in which $S$ is multiplicatively closed, need not be a $D$-domain.

EXAMPLE 1. Let $R=F[x]$ be the polynomial ring over the totally ordered field $F$, and let $R^{+}=\left\{\alpha_{0}+\alpha_{1} x+\cdots+\alpha_{n} x^{n}: \alpha_{0} \geqq 0\right.$ and $\left.\alpha_{n} \geqq 0\right\}$. As an $l$-group $R$ is the direct sum of two totally ordered groups, and so it has only two roots. Its set of special elements is

$$
S=\{\alpha: 0<\alpha \in F\} \cup\left\{\alpha_{1} x+\cdots+\alpha_{n} x^{n}: \alpha_{n}>0\right\} .
$$

Since $1 \wedge x=0$, but $x \wedge x^{2}>0, R$ is not a $D$-domain.

For the remainder of this paper let $R$ be a $D$-domain with special elements $S$. By Proposition 2, $S$ is a multiplicatively closed subset of $R$. If $Q$ is the ring of quotients of $R$ with respect to $S$, then Proposition 1 says that $Q$ is an $l$-ring extension of $R$. Let $T$ be the set of special elements of $Q$.

Proposition 3. $T$ is the quotient po-group of $S$; i.e., $T=\left\{a S^{-1} \in Q: a\right.$, $s \in S\}$.

Proof. If $a, s \in S$, then $\left(a s^{-1}\right)^{-1}=s a^{-1} \in Q^{+}$. So $a s^{-1} \in T$, by the corollary to Lemma 2. Conversely, suppose that $a s^{-1} \in T$. By Lemma 2(a), $a \in T$. Let $Q(a)$ (respectively $R(a)$ ) be the convex $l$-subgroup of $Q$ (respectively $R$ ) generated by $a$. Then $Q(a)=\operatorname{lex} N$ for a proper convex $l$-subgroup $N$ of $Q(a)$ [2, Theorem 3.6]. But then $N \cap R(a) \subsetneq R(a)$, and $R(a)=\operatorname{lex}(N \cap R(a))$. Thus $a \in S$.

Corollary 1. $Q$ is a $D$-domain and $T=\left\{q \in Q^{+}: q^{-1} \in Q^{+}\right\}$.

Proof. This follows immediately from Proposition 3 and the previous corollary.

Note that $T \cap R=S$ and that $S$ is actually the set of basic elements of $R$.

COROLlary 2. $R$ is finitely-valued if and only if its underlying l-group is a direct sum of totally ordered groups.

PROof. The only if part follows from the fact that each special element is basic and from [8, Theorem 2.12]. The if part is trivial.

Proposition 4. (a) If $R$ is finitely-valued, then so is $Q$.

(b) $R$ has exactly $n$ roots if and only if $Q$ has exactly $n$ roots. 
Proof. Suppose that $R$ is finitely-valued and $g s^{-1} \in Q^{+}$. Then $g$ is the sum of pairwise disjoint special elements of $R ; g=g_{1}+\cdots+g_{n}$ [2, Theorem 3.7]. Thus $g s^{-1}$ is the sum of pairwise disjoint special elements of $Q$. So $Q$ is finitely-valued.

As is well known $R$ has exactly $n$ roots if and only if $R$ has a basis containing exactly $n$ elements [5]. Suppose that $R$ has exactly $n$ roots and $\left\{g_{i} u_{i}^{-1}: i=1, \cdots, m\right\}$ is a set of pairwise disjoint elements of $Q$. Let $s_{i}=u_{1} \cdots u_{i-1} u_{i+1} \cdots u_{m}$. Then $\left\{g_{i} s_{i}: i=1, \cdots, m\right\}$ is a set of pairwise disjoint elements of $R$. So $m \leqq n$, and $Q$ has at most $n$ roots. But $R$ contains $n$ disjoint elements, so $Q$ has at least $n$ roots. Thus $Q$ has exactly $n$ roots.

Conversely, if $\left\{g_{i} s^{-1}: i=1, \cdots, n\right\}$ is a basis of $Q$, then $\left\{g_{i}: i=1, \cdots, n\right\} \subset$ $S$. Since $R$ cannot have more than $n$ disjoint elements, $\left\{g_{i}: i=1, \cdots, n\right\}$ is a basis of $R$.

Proposition 5. Each D-domain contains a unique largest convex $l$ subring that is a finitely-valued D-domain.

Proof. We first show that the sum of a finitely-rooted convex $l$ subgroup $A$ and a totally ordered subgroup $B$ of an $l$-group $G$ is a finitelyrooted $l$-subgroup. For $A+B$ is an $l$-subgroup of $G$ since $B+A / A$ is one of $G / A$. Note that since $B+A|A \cong B| A \cap B, A$ is a prime subgroup of $B+A$. Suppose that $A$ has only $n$ roots, and let $\left\{a_{i}+b_{i}: a_{i} \in A, b_{i} \in B ; i=1, \cdots, m\right\}$ be $m$ disjoint elements in $A+B$. If $b_{i} \in A$ for all $i$, then clearly $m \leqq n$. If $b_{i} \notin A$ for some $i$, then $a_{j}+b_{j} \in A$ for $j \neq i$, and so $m \leqq n+1$. Thus $A+B$ is finitely-rooted. By induction, it is easily seen that the sum of $n$ totally ordered convex $l$-subgroups of an $l$-group has at most $n$ roots.

Now let $S$ be the set of special elements of the $D$-domain $R$, and let $A$ be the additive subgroup of $R$ generated by $S$ :

$$
A=\left\{g_{1}+\cdots+g_{n}:\left|g_{i}\right| \in S\right\} .
$$

Since each special element is basic, $g \in S$ implies $C(g) \subseteq A$. So, by the preceding paragraph, $A$ is a convex $l$-subring of $R$. Since each special element of $A$ is special in $R$ [2, Theorem 3.5], $A$ is a $D$-domain. It is clearly the largest convex $l$-sub- $D$-domain of $R$ that is finitely-valued.

Note that Example 2 and the remarks following it show that the finitelyvalued part of a $D$-field need not be a field.

If $\Gamma$ is the value set of $R$, then the mapping $v_{R}: S \rightarrow \Gamma$ that sends each element of $S$ to its value is order preserving. Moreover, $v_{R}$ has the following properties:

(1) $v_{R}(s)=v_{R}(t)$ if and only if $C(s)=C(t)$.

(2) $v_{R}(s)<v_{R}(t)$ if and only if $s$ is infinitely smaller than $t$.

(3) If $v_{R}(s)=v_{R}(t)$ and $a \in S$, then $v_{R}(a s)=v_{R}(a t)$. 
The last property implies that the multiplication in $S$ can be transferred to $v_{R}(S)$ via $v_{R}$. Thus, using additive notation, $v_{R}(S)$ becomes a rooted partially-ordered semigroup if addition is defined by $v_{R}(s)+v_{R}(t)=v_{R}(s t)$. We will say that $v_{R}(S)$ is torsion-free if for all $\alpha, \beta \in v_{R}(S)$ and $0 \neq n \in Z^{+}$, $n \alpha=n \beta$ implies $\alpha=\beta$.

We can, of course, do the same thing for $Q$. In this case $v_{Q}(T)$ becomes a rooted po-group since $T$ is a group. It is clear that $v_{Q}(T)$ is the quotient po-group of $v_{R}(S)$, where $v_{R}(S)$ is embedded in $v_{Q}(T)$ via $v_{R}(a) \rightarrow v_{Q}(a)$. Note that $v_{Q}(T)$ is a torsion-free group exactly when $v_{R}(S)$ is a torsion-free semigroup. For brevity, we will call $\left(Q, T, v_{Q}(T)\right)$ the quotient system of $\left(R, S, v_{R}(S)\right)$.

Now suppose that $R$ is finitely-valued. Then $v_{R}(S)=\Gamma$ [2, Theorem 3.8]. By Proposition 4, $Q$ is also finitely-valued; so $v_{Q}(T)=\Delta$ is the value set of $Q$. We now state the main results of this paper. As mentioned earlier, their proofs are identical with those given in [3] for the case that $R$ is a field. $V(\Gamma, R)$ is the formal power series $l$-ring with exponents in $\Gamma$, coefficients in the real field $\boldsymbol{R}$, and whose lattice order is that of its underlying Hahn product.

THEOREM 1. Let $R$ be a finitely-valued $D$-domain whose value semigroup $\Gamma$ is torsion-free. Then the lattice order of $R$ can be extended to a total (ring) order of $R$.

THEOREM 2. Let $R$ be a finitely-rooted $D$-domain, and let $(Q, T, \Delta)$ be the quotient system of $(R, S, \Gamma)$. If $\Gamma$ is torsion-free there is a value preserving l-isomorphism of $Q$ into the l-field $V(\Delta, R)$, whose restriction to $R$ is also value preserving.

3. Remarks. The following example shows that the quotient $l$-ring $Q$ of a finitely-valued $D$-domain $R$ (for which $\Gamma$ is torsion-free) need not be its quotient field. Note that any such $Q$ must be $l$-simple, i.e., 0 and $Q$ are its only $l$-ideals. If $Q$ is finitely-rooted we do not know if it must be a field.

EXAMPLE 2. Let $R=F[x]$ be the polynomial ring over the totally ordered field $F$. $R$ becomes a $D$-domain if its positive cone is defined by $R^{+}=\left\{\sum_{i=0}^{n} \alpha_{i} x^{i}: \alpha_{i} \geqq 0\right.$ for each $\left.i\right\}$. Then $S=\left\{\alpha x^{n}: \alpha>0\right\}, Q=\left\{\sum_{i=-m}^{n} \alpha_{i} x^{i}\right\}$, $Q^{+}=\left\{\sum_{i=-m}^{n} \alpha_{i} x^{i}: \alpha_{i} \geqq 0\right\}$, and $T=\left\{\alpha x^{n}: n \in Z\right.$ and $\left.\alpha>0\right\}$. Using (1) and (2) it is easily seen that $\Gamma \cong v(F) \oplus Z^{+}$and $\Delta \cong v(F) \oplus Z$, where $v(F)$ is the value group of $F$ and $Z\left(Z^{+}\right)$is the trivially ordered group (semigroup) of (positive) integers.

We have not been able to determine whether the quotient field $E$ of a $D$-domain $R$ can be made into an $l$-ring extension of $R$. In the preceding example $Q$ can be value-embedded in an $l$-field $G$ that is a $D$-domain, but 
which is not finitely-valued:

$$
G=\left\{\sum_{i=-\infty}^{n} \alpha_{i} x^{i}: \alpha_{i} \in F \text { and } n \in Z\right\}
$$

and

$$
G^{+}=\left\{\sum_{i=-\infty}^{n} \alpha_{i} x^{i}: \alpha_{i} \geqq 0 \text { for each } i\right\} .
$$

If $R$ is finitely-rooted and $\Delta$ is torsion-free, and if $E$ is an $l$-subring of $V(\Delta, \boldsymbol{R})$, then $E$ is a $D$-domain.

Examples of $D$-domains may be obtained as follows. Let $\Delta$ be a finitelyrooted torsion-free abelian po-group, and let $\Delta_{1}$ be the unique totally ordered group whose underlying group is $\Delta$ and such that $\Delta^{+} \subseteq \Delta_{1}^{+}[3$, p. 387]. Let $V=V(\Delta, R)$ and $V_{1}=V\left(\Delta_{1}, R\right)$. Then $V$ and $V_{1}$ are the same ring, and $V^{+} \subseteq V_{1}^{+}[3,2.2]$. Let

$$
\begin{aligned}
R & =\left\{g \in V_{1}: \text { value of } g \leqq 0\right\} \cup\{0\} \\
& =\left\{g \in V_{1}:|g| \leqq n \text { for some positive integer } n\right\} .
\end{aligned}
$$

Then $R$ is a sub- $D$-domain of $V$, and $Q(R)=V$. If $\Delta$ is not abelian, then $R$ is a noncommutative $D$-domain.

Finally, we note that the results of $\S 2$ hold if $R$ is only a commutative $l$-domain (but otherwise satisfying the defining properties of a $D$-domain). For the proof of Proposition 2 remains valid provided $S_{1}$ consists of regular elements. But if $a \in S_{1}$, then $a b=0$ implies $a b^{+}=a b^{-}=0$. Hence $b=0$, and $a$ is regular. Now Propositions 3 through 5 hold for $R$; and the proof of Theorem 1 shows that if $R$ is finitely-valued and $\Gamma$ is torsion-free, then $R$ is, in fact, a domain.

ADDED IN PROOF. Professor Henriksen has informed us that the quotient field of a finitely-valued $D$-domain $R$ cannot always be made into an $l$ ring extension of $R$.

\section{REFERENCES}

1. F. W. Anderson, Lattice-ordered rings of quotients, Canad. J. Math. 17 (1965), 434-448. MR 30 \#4801.

2. P. Conrad, The lattice of all convex l-subgroups of a lattice-ordered group, Czechoslovak Math. J. 15 (90) (1965), 101-123. MR 30 \#3926.

3. P. Conrad and J. Dauns, An embedding theorem for lattice-ordered fields, Pacific J. Math. 30 (1969), 385-398. MR 40 \#128.

4. P. Conrad and J. E. Diem, The ring of polar preserving endomorphisms of an abelian lattice-ordered group, Illinois J. Math. 15 (1971), 222-240.

5. P. Conrad, J. Harvey and C. Holland, The Hahn embedding theorem for abelian lattice-ordered groups, Trans. Amer. Math. Soc. 108 (1963), 143-169. MR 27 \#1519. 
6. J. E. Diem, A radical for lattice-ordered rings, Pacific J. Math. 25 (1968), 71-82. MR 37 \#2653.

7. L. Fuchs, Teilweise geordnete algebraische Strukturen, Studia MathematicaMathematische Lehrbücher, Band 19, Vandenhoeck and Ruprecht, Göttingen, 1966. MR 34 \#4386.

8. S. A. Steinberg, Finitely-valued f-modules, Pacific J. Math. (to appear).

9. E. C. Weinberg, Lectures on ordered groups and rings, Lecture Notes, University of Illinois, Urbana, Illinois, 1968.

Department of Mathematics, University of Missouri-St. Louis, St. Louis, MISSOURI 63121

Current address: Department of Mathematics, University of Toledo, Toledo, Ohio 43606 